\title{
A BOUNDARY VALUE PROBLEM FOR A SYSTEM OF ORDINARY LINEAR DIFFERENTIAL EQUATIONS OF THE FIRST ORDER*
}

\author{
BY \\ GILBERT AMES BLISS
}

The boundary value problem to be considered in this paper is that of finding solutions of the system of differential equations and boundary conditions

$$
\begin{aligned}
& \frac{d y_{i}}{d x}=\sum_{\alpha=1}^{n}\left[A_{i \alpha}(x)+\lambda B_{i \alpha}(x)\right] y_{\alpha}(x), \\
& \sum_{\alpha=1}^{n}\left[M_{i \alpha} y_{\alpha}(a)+N_{i \alpha} y_{\alpha}(b)\right]=0 \quad(i=1,2, \cdots, n) .
\end{aligned}
$$

Such systems have been studied by a number of writers whose papers are cited in a list at the close of this memoir. The further details of references incompletely given in the footnotes or text of the following pages will be found there in full.

In 1909 Bounitzky defined for the first time a boundary value problem adjoint to the one described above, and discussed its relationships with the original problem. He constructed the Green's matrices for the two problems, and secured expansion theorems by considering the system of linear integral equations, each in one unknown function, whose kernels are the elements of the Green's matrix. In 1918 Hildebrandt, following the methods of $\mathbf{E}$. $\mathbf{H}$. Moore's general analysis, formulated a very general boundary value problem containing the one above as a special case, and established a number of fundamental theorems. In $1921 \mathrm{~W}$. A. Hurwitz studied the more special system

$$
\begin{array}{ll}
\frac{d u}{d x}=[a(x)+\lambda] v(x), & \frac{d v}{d x}=-[b(x)+\lambda] u(x), \\
\alpha_{0} u(0)+\beta_{0} v(0)=0, & \alpha_{1} u(1)+\beta_{1} v(1)=0
\end{array}
$$

and its expansion theorems, by the method of asymptotic expansions, and in 1922 Camp extended his results to a case where the boundary conditions have a less special form. Meanwhile Schur in 1921 published very general expansion theorems for the original system (1) under the hypothesis that the matrix of functions $B_{i k}(x)$ has all elements identically zero except those

\footnotetext{
* Presented to the Society, December 30, 1924; received by the editors in December, 1925.
} 
in the principal diagonal, which are real, distinct, and positive for every value of $x$. The cases of Hurwitz and Camp are not included under this one even after a linear transformation. Carmichael exhibited in 192122 the analogies between certain algebraic problems and boundary value problems of many typis, including those of the type (1), but without giving the details of the theory of the boundary value problems themselves. In 1923 Birkhoff and Langer showed that the large class of systems (1) for which the determinant of the functions $B_{i k}(x)$ is different from zero can be reduced, by a linear transformation of the functions $y_{k}(x)$ whose coefficients may be imaginary, to the simpler form for which all the functions $B_{i k}(x)$ with $i \neq k$ vanish identically. They obtained expansion theorems under the further hypotheses that the functions $B_{i i}(x)$ are all distinct from each other and from zero, though not necessarily real and positive as in Schur's paper, and that they satisfy certain other more artificial restrictions.* The problems considered by Hurwitz, Camp, and Schur are among those included in their theory. An abstract of the paper of Birkhoff and Langer was printed in $1922 . \dagger$

The methods of Hurwitz, Camp, Schur, and Birkhoff and Langer are those of asymptotic expansions which for the more general cases become very complicated. In the following pages it will be shown that a large class of so called self-adjoint boundary value problems, analogous to equations with symmetric kernels in linear integral equation theory, can be treated by much more elementary methods.

A boundary value problem adjoint to the problem (1) has the form

$$
\begin{aligned}
& \frac{d z_{i}}{d x}=-\sum_{\alpha=1}^{n}\left(A_{\alpha i}+\lambda B_{\alpha i}\right) z_{\alpha}, \\
& \sum_{\alpha=1}^{n}\left[P_{\alpha i} z_{\alpha}(a)+Q_{\alpha i} z_{\alpha}(b)\right]=0 \quad(i=1,2, \cdots, n),
\end{aligned}
$$

where the coefficients $P_{i k}, Q_{i k}$ satisfy the relations

$$
M_{i x} P_{\alpha k}-N_{i \alpha} Q_{\alpha k}=0 \quad(i, k=1,2, \cdots, n) .
$$

The original problem is defined in this paper to be self-adjoint if the systems (1) and (3) are equivalent under a transformation of the form

$$
z_{i}=\sum_{\alpha=1}^{n} T_{i \alpha}(x) y_{\alpha}
$$

- See pp. 83, 89, 109. One should note also the hypotheses on the roots of $D(\lambda)$ on pp. 98 and 105.

† See also an earlier abstract by Birkhoff, Bulletin of the American Mathematical Society, vol. 25 (1919), p. 442. 
and it is definitely self-acijoint when a further hypothesis, explained in Section 2 , is added. For a definitely self-adjoint boundary value problem the characteristic constants and functions are not only denumerably infinite in number, but the constants are real and each has its index equal to its multiplicity as a root of the characteristic determinant. The characteristic functions may be chosen real. Furthermore expansion theorems of a very general sort may be justified by relatively simple methods analogous to those of integral equation theory.

The problems of Hurwitz and Camp described above are definitely selfadjoint according to the definition of this paper. Those of Schur are never self-adjoint, and a sub-class only of the problems considered by Birkhoff and Langer have this property. The problems of Birkhoff and Langer are furthermore never definitely self-adjoint when the coefficients in the differential equations are real. On the other hand, the theory of definitely selfadjoint boundary value problems as presented here includes a large category of problems for which the determinant $\left|B_{i k}(x)\right|$ vanishes and which do not fall under any of the cases treated by the authors just referred to. In this category are problems of the type (1) which arise in connection with the calculus of variations, all of which have the property of self-adjointness described above. The importance of this class is evident when one considers the fact that the boundary problems of mathematical physics almost invariably belong to it. Another case is the system of the type (1) equivalent to the well known boundary value problem for a single linear differential equation of the $n$th order. This system does not fall under the theories presented in the papers mentioned above, and only self-adjoint equations of this sort give rise to self-adjoint problems of the type considered in the following pages. The interrelationships just mentioned between problems of various types are not elucidated in detail in this paper. I expect to return to them on another occasion.

The properties of the system (1) which justify expansion theorems analogous to those well known for other problems have not so far been clearly classified and analyzed. They seem to depend primarily upon the character of the matrix of functions $B_{i k}(x)$. A further study of this question, and a correlation of the methods and results hitherto attained, is desirable.

The methods used in the following pages are developed independently of the theory of linear integral equations though there are many points of contact. In Section 4 below it is shown that the equations (1) are equivalent to a certain system of linear integral equations for the functions $y_{i}(x)$, a result which is well known. The kernel matrix of these integral equations is not in general symmetric. When (1) is self-adjoint, however, every solution 
$y_{i}(x)$ of the integral equations goes into a solution of the "associated" system of integral equations by means of a transformation of the form

$$
u_{i}=\sum_{\alpha=1}^{n} S_{i \alpha} y_{\alpha}=\sum_{\alpha=1}^{n} \sum_{\beta=1}^{n} B_{\beta i} T_{\beta \alpha} y_{\alpha}
$$

for which the matrix $\left\|S_{i k}\right\|$ is symmetric. If the determinant $\left|B_{i k}\right|$ is different from zero the same is true of $\left|S_{i k}\right|$, and the system of integral equations equivalent to (1) can be reduced to one with a symmetric kernel matrix by means of a suitable transformation. The theory of the boundary value problem is then a corollary to the theory of a system of integral equations with symmetric kernel matrix such as has been developed, for example, by E. H. Moore in his "general analysis." The case when the determinant $\left|B_{i k}\right|$ vanishes includes some of the most important boundary value problems, as has been explained in the preceding paragraphs. For some of these it is possible to reduce the number of functions $y_{i}(x)$ by transformation and thereby to change the problem into an equivalent one whose corresponding integral equations have a symmetric kernel matrix, but it does not seem possible always to make such a transformation.

1. Adjoint systems. In the following pages the notations of tensor analysis will be used. It is understood that the indices $i, j, k, \cdots, \alpha, \beta$, $\boldsymbol{\gamma}, \cdots$ have the range $1,2, \cdots, n$ and that an expression such as $A_{i \alpha} B_{k \alpha}$ stands for the sum

$$
A_{i \alpha} B_{k \alpha}=A_{i 1} B_{k 1}+\cdots+A_{i n} B_{k n}
$$

taken with respect to the repeated index $\alpha$. The index $\alpha$ may be called an umbral index.* Such indices will usually be denoted by Greek letters.

As a matter of formal algebra, consider the four linear expressions

$$
\begin{array}{ll}
s_{i}(y)=M_{i \alpha} y_{\alpha}(a)+N_{i \alpha} y_{\alpha}(b), & t_{i}(z)=P_{\alpha i} z_{\alpha}(a)+Q_{\alpha i} z_{\alpha}(b), \\
\bar{s}_{i}(y)=\bar{M}_{i \alpha} y_{\alpha}(a)+\bar{N}_{i \alpha} y_{\alpha}(b), & \bar{t}_{i}(z)=\bar{P}_{\alpha i z_{\alpha}}(a)+\bar{Q}_{\alpha i z_{\alpha}}(b),
\end{array}
$$

formed for two sets of functions $y_{i}(x), z_{i}(x)$. The coefficients in these expressions are real constants and the symbols on the left are merely notations for the sums on the right. If the coefficients in the sums $s_{i}(y)$ are given, with matrix of rank $n$, then a matrix of coefficients for $t_{i}(z)$ can always be selected also of rank $n$ and so that the relations

$$
M_{i \alpha} P_{\alpha k}-N_{i \alpha} Q_{\alpha k}=0
$$

\footnotetext{
- Murnaghan, Vector Analysis and the Theory of Relativity, p. 7.
} 
are satisfied. The coefficients of the auxiliary sums $\bar{s}_{i}(y)$ and $\bar{t}_{i}(z)$ can then be chosen so that the two matrices

$$
\left\|\begin{array}{ll}
M_{i k k} & N_{i k} \\
\bar{M}_{i k} & \bar{N}_{i k}
\end{array}\right\| \quad\left\|\begin{array}{rr}
-\bar{P}_{i k} & -P_{i k} \\
\bar{Q}_{i k} & Q_{i k}
\end{array}\right\|,
$$

with $i$ constant in the rows and $k$ in the columns, are reciprocals. From the relationships between the coefficients of these matrices found by multiplying them in reverse order it follows readily that the equation

$$
s_{\alpha}(y) \bar{t}_{\alpha}(z)+\bar{s}_{\alpha}(y) t_{\alpha}(z)=\left.y_{\alpha}(x) z_{\alpha}(x)\right|_{a} ^{b}
$$

is an identity for all systems $y_{i}(x), z_{i}(x)$. This is an equation which will be frequently useful. When the coefficients of the sums $s_{i}(y)$ are given the coefficients of the $t_{i}(z)$, chosen as above indicated, form $2 n$ linearly independent solutions $u_{i}, v_{i}$ of the equations

$$
M_{i \alpha} u_{\alpha}-N_{i \alpha} v_{\alpha}=0 .
$$

All other solutions of these equations are expressible linearly in terms of these $n$, and it follows readily that two different choices of the coefficients in $t_{i}(z)$ give systems of equations $t_{i}(z)=0$ which are equivalent.

The systems of differential equations and boundary conditions considered in this paper may be written in the form

$$
y_{i}^{\prime}(x)=A_{i \alpha}(x) y_{\alpha}(x), \quad s_{i}(y)=M_{i \alpha} y_{\alpha}(a)+N_{i \alpha} y_{\alpha}(b)=0 .
$$

The coefficients $A_{i k}(x)$ are supposed to be real single-valued continuous functions on the interval $a \leqq x \leqq b$; the matrix $\left\|M_{i k}, N_{i k}\right\|$ of coefficients in $s_{i}(y)$ is of rank $n$; and solutions $y_{i}(x)$ of the equations, continuous with their derivatives on the interval $a b$, are sought. A system adjoint to (8) is by definition one of the form

$$
z_{i}^{\prime}(x)=-A_{\alpha i}(x) z_{\alpha}(x), \quad t_{i}(z)=P_{\alpha i} z_{\alpha}(a)+Q_{\alpha i} z_{\alpha}(b)=0
$$

where $t_{i}(z)$ has been formed from $s_{i}(y)$ in the manner described in the preceding paragraph.*

Let $\left\|Y_{i k}(x)\right\|$ be a matrix of functions whose columns form $n$ linearly independent solutions of the differential equations in the system (8). The most general solution of these equations has the form $y_{i}(x)=Y_{i \alpha} c_{\alpha}$ where the coefficients $c_{i}$ are constants. The solution $Y_{1 i}, \cdots, Y_{n i}$ will be denoted simply by $Y_{i}$ when no confusion results, and the determinant whose elements

- This definition is that of Bounitzky, p. 73, and is analogous to that of Birkhoff for linear equations of the $n$th order, these Transactions, vol. 9 (1908), p. 375. 
are $D_{i k}=s_{i}\left(Y_{k}\right)$ will be denoted by $D$. For the adjoint system (9) the corresponding notations will be $Z_{i k}, E$. The first two theorems to be proved below are analogous to similar theorems for linear integral equations and for other types of boundary value problems, and are already in the literature.*

THEOREM 1. If the determinant $D$ is different from zero the only solution of the boundary value problem $(8)$ is $y_{i}(x)=0$. If $D$ has rank $n-r$ then the problem has $r$ and only $r$ linearly independent non-vanishing solutions.

This is easy to see, since every solution of the boundary value problem must have the form $y_{i}=Y_{i \alpha} c_{\alpha}$ with coefficients $c_{i}$ satisfying the linear equations $D_{i \alpha} c_{\alpha}=s_{i}\left(Y_{\alpha}\right) c_{\alpha}=0$.

THEOREM 2. The number of linearly independent non-vanishing solutions of the original system (8) is always the same as the number for the adjoint system (9).

To prove this one may note first that for every pair $y_{i}(x), z_{i}(x)$ of solutions of the differential equations in (8) and (9) the equations

$$
\begin{gathered}
z_{\beta} y_{\beta}^{\prime}+z_{\beta}^{\prime} y_{\beta}=A_{\beta \alpha} y_{\alpha} z_{\beta}-A_{\alpha \beta} y_{\beta} z_{\alpha}=0, \\
\left.y_{\alpha}(x) z_{\alpha}(x)\right|_{a} ^{b}=0
\end{gathered}
$$

hold true. Suppose then that $U_{1 p}, \cdots, U_{n p}(p=1,2, \cdots, r)$ are $r$ linearly independent solutions of the boundary value problem (8). The $r \operatorname{sets} \bar{s}_{i}\left(U_{p}\right)$ $(p=1,2, \cdots, r)$ are also linearly independent, since otherwise there would be a set of functions $y_{i}=U_{i p} c_{p}$, with constants $c_{p}$ not all zero, making the expressions $\bar{s}_{i}(y)$, as well as the sums $s_{i}(y)$, all zero. Since the determinant of the first matrix (6) is not zero the equations (4) would then imply $y_{i}(a)=$ $y_{i}(b)=0$, and the solution $y_{i}(x)$ would have to vanish identically, which is impossible when the solutions $U_{p}$ are linearly independent. From the relations (7) and (10) it follows now that

$$
\bar{s}_{\alpha}\left(U_{p}\right) t_{\alpha}\left(Z_{k}\right)=0 \quad(k=1,2, \cdots, n ; p=1,2, \cdots, r) .
$$

Hence the determinant $E$ whose elements are $E_{i k}=t_{i}\left(Z_{k}\right)$ has at most rank $n-r$. By similar reasoning it follows that when $E$ has rank $n-r$ the rank of $D$ is at most $n-r$, so that $D$ and $E$ have the same rank, and the number of linearly independent solutions of the systems (8) and (9) is the same.

THEOREM 3. If the determinant $D$ is different from zero the non-homogeneous system

$$
y_{i}^{\prime}(x)=A_{i \alpha}(x) y_{\alpha}(x)+g_{i}(x), \quad s_{i}(y)=h_{i},
$$

- Bounitzky, p. 77; Birkhoff-Langer, p. 64-5; Hurwitz, p. 526; Camp, p. 30. 
where the functions $g_{i}(x)$ are continuous on the interval $a b$, has one and only one solution.

The differential equations of the system (11) have as their general solution

$$
y_{i}(x)=Y_{i 0}(x)+Y_{i \alpha}(x) c_{\alpha}
$$

where $Y_{0}$ is a particular solution and the sets $Y_{\alpha}$ are as before linearly independent solutions of the equations with $g_{i}(x)=0$. Since $D=\left|s_{i}\left(Y_{k}\right)\right|$ is different from zero the constants $c_{i}$ can be chosen in one and but one way so that

$$
s_{i}(y)=s_{i}\left(Y_{0}\right)+s_{i}\left(Y_{\alpha}\right) c_{\alpha}=h_{i}
$$

TheORem 4. If the determinant $D$ has rank $n-r$ then the system (11) has solutions if and only if the equation

$$
\int_{a}^{b} z_{\alpha}(x) g_{\alpha}(x) d x=0
$$

is satisfied for every solution $z_{i}(x)$ of the adjoint system (9). The most general solution of (11) is then

$$
y_{i}(x)=y_{i}^{*}(x)+c_{1} U_{i 1}(x)+\cdots+c_{r} U_{i r}(x)
$$

where $y_{i}^{*}(x)$ is a particular solution and the sets $U_{i p}(p=1, \cdots, r)$ are $r$ linearly independent solutions of the original system (8).

If $y_{i}(x)$ and $z_{i}(x)$ are solutions of the differential equations in the systems (11) and (9), then one readily verifies that

$$
y_{\alpha}^{\prime} z_{\alpha}+y_{\alpha} z_{\alpha}^{\prime}=g_{\alpha} z_{\alpha},
$$

and hence, with the help of equations (7), that

$$
\int_{a}^{b} g_{\alpha} z_{\alpha} d x=\left.y_{\alpha} z_{\alpha}\right|_{a} ^{b}=s_{\alpha}(y) \bar{t}_{\alpha}(z)+\bar{s}_{\alpha}(y) t_{\alpha}(z) .
$$

If $y$ and $z$ satisfy the boundary conditions in (11) and (9) the value of this expression is zero.

Suppose, conversely, that equation (13) holds true for every solution $z_{i}(x)$ of the adjoint system (9). Every solution of the original system (11) must have the form (12) with constants $c_{i}$ satisfying the equations

$$
s_{i}(y)=s_{i}\left(Y_{0}\right)+c_{\alpha} s_{i}\left(Y_{\alpha}\right)=0 .
$$

By the argument of the proof of Theorem 2 it follows that the sets $\bar{t}_{i}(z)$, formed for $r$ linearly independent solutions $z_{i}(x)$ of the adjoint system (9), 
are themselves $r$ linearly independent solutions of the linear equations whose coefficients are the columns of the determinant $D=\left|s_{i}\left(Y_{k}\right)\right|$. Hence the last equations have solutions $c_{i}$ if and only if the conditions $\bar{t}_{\alpha}(z) s_{\alpha}\left(Y_{0}\right)=0$ are satisfied for every solution $z_{i}(x)$ of the adjoint system (9). But from equation (15) with $y$ replaced by $Y_{0}$, and from the boundary conditions $t_{i}(z)=0$, it follows then that solutions $c_{i}$ will certainly exist since the conditions

$$
0=\int_{a}^{b} g_{\alpha} z_{\alpha} d x=s_{\alpha}\left(Y_{0}\right) t_{\alpha}(z)
$$

are satisfied.

The last statement of the theorem is true since if $y_{i}(x)$ and $y_{i}{ }^{*}(x)$ are both solutions of the non-homogeneous system (11), their difference $y_{i}(x)$ $-y_{i}^{*}(x)$ satisfies the original homogeneous system (8).

2. Self-adjoint systems. Consider now a system of the form

$$
y_{i}^{\prime}=\left(A_{i \alpha}+\lambda B_{i \alpha}\right) y_{\alpha}, \quad s_{i}(y)=M_{i \alpha} y_{\alpha}(a)+N_{i \alpha} y_{\alpha}(b)=0
$$

containing a parameter $\lambda$ linearly and having its coefficients $A_{i k}(x), B_{i k}(x)$ all continuous on the interval $a \leqq x \leqq b$. The functions $B_{i k}(x)$ are by hypothesis not all identically zero. An adjoint system has the form

$$
z_{i}^{\prime}=-\left(A_{\alpha i}+\lambda B_{\alpha i}\right) z_{\alpha}, \quad t_{i}(z)=P_{\alpha i} z_{\alpha}(a)+Q_{\alpha i} z_{\alpha}(b)=0,
$$

where $s_{i}(y)$ and $t_{i}(z)$ are related as in the preceding section.

The existence theorems for differential equations tell us that there exists a matrix $\left\|Y_{i k}(x, \lambda)\right\|$ whose columns are $n$ linearly independent solutions of the differential equations in the system (16) and whose elements with their derivatives with respect to $x$ are expressible as permanently convergent power series in $\lambda$. The determinant $D(\lambda)$ formed for this system is therefore also representable by a permanently convergent power series. The roots of $D(\lambda)$ are called the characteristic values of the parameter $\lambda$, and the nonvanishing solutions $y_{i}(x)$ of the system (16) corresponding to such values are called characteristic solutions. The corresponding notations for the adjoint system (17) are $Z_{i k}(x, \lambda)$ and $E(\lambda)$. It is well known that the roots of a permanently convergent power series $D(\lambda)$ are finite or at most denumerably infinite in number. The following theorem is an immediate consequence of Theorems 1 and 2 of the preceding section.

THEOREM 5. The characteristic parameter values for the boundary value problem (16) are identical with those for the adjoint problem (17). The number of linearly independent characteristic solutions corresponding to a particular characteristic value $\lambda$ is the same for both problems. 
THEOREM 6. If $\lambda_{1} \neq \lambda_{2}$ are characteristic values and $y_{i}(x), z_{i}(x)$ corresponding characteristic solutions of (16) and (17), then

$$
\int_{a}^{b} B_{\alpha \beta}(x) y_{\alpha}(x) z_{\beta}(x) d x=0 \text {. }
$$

For from the differential equations in (16) and (17) with $\lambda_{1}$ and $\lambda_{2}$ substituted it follows that

$$
y_{\alpha}^{\prime} z_{\alpha}+y_{\alpha} z_{\alpha}^{\prime}=\lambda_{1} B_{\alpha \beta} y_{\beta} z_{\alpha}-\lambda_{2} B_{\beta \alpha} y_{\alpha} z_{\beta},
$$

and hence, from the boundary conditions $s_{i}(y)=t_{i}(z)=0$ and equation (7), that

$$
0=\left.y_{\alpha} z_{\beta}\right|_{a} ^{b}=\left(\lambda_{1}-\lambda_{2}\right) \int_{a}^{b} B_{\beta \alpha} y_{\alpha} z_{\beta} d x
$$

In the following pages a set of relations of the form $z_{i}=T_{i \alpha}(x) y_{\alpha}$ will be called a transformation if the functions $T_{i k}(x)$ are real, single-valued, and have continuous derivatives on the interval $a b$, and if the determinant $\left|T_{i k}(x)\right|$ is different from zero on that interval. The coefficients of the inverse transformation will be denoted by $T_{\mathfrak{i k}}^{-1}(x)$.

Definition of a self-adjoint system. The boundary value problem (16) is said to be self-adjoint if the differential equations and also the boundary conditions of its adjoint (17) are equivalent to its own for all values of $\boldsymbol{\lambda}$ by means of a transformation $z_{i}=T_{i \alpha}(x) y_{\alpha}$.

THEOREM 7. In order that the problem (16) shall be self-adjoint it is necessary and sufficient that there shall exist a transformation $T_{i k}(x)$ such that

$$
\begin{gathered}
T_{i \alpha} A_{\alpha k}+A_{\alpha i} T_{\alpha k}+T_{i k}^{\prime} \equiv 0, \quad T_{i \alpha} B_{\alpha k}+B_{\alpha i} T_{\alpha k} \equiv 0, \\
M_{i \alpha} T_{\alpha \beta}^{-1}(a) M_{k \beta}=N_{i \alpha} T_{\alpha \beta}^{-1}(b) N_{k \beta} .
\end{gathered}
$$

To prove the first two of these relations one can verify readily that the transformation $z_{i}=T_{i \alpha} y_{\alpha}$ takes the differential equations of the system (16) into the set

$$
z_{i}^{\prime}=\left[T_{i \alpha} A_{\alpha \beta} T_{\beta \gamma}^{-1}+\lambda T_{i \alpha} B_{\alpha \beta} T_{\beta \gamma}^{-1}+T_{i \beta}^{\prime} T_{\beta \gamma}^{-1} z_{\gamma} .\right.
$$

If these are equivalent to the differential equations in the adjoint system (17) for all values of $\lambda$, the equations (19) follow at once.

By the transformation $z_{i}=T_{i \alpha} y_{\alpha}$ the boundary conditions of the adjoint problem take the form

$$
P_{\alpha i} T_{\alpha \beta}(a) y_{\beta}(a)+Q_{\alpha i} T_{\alpha \beta}(b) y_{\beta}(b)=0 .
$$


For these to be equivalent to the original boundary conditions $s_{i}(y)=0$ it is necessary and sufficient that a matrix of constants $C_{i k}$ with determinant different from zero exists such that

$$
P_{\alpha i} T_{\alpha k}(a)=C_{i \alpha} M_{\alpha k}, \quad Q_{\alpha i} T_{\alpha k}(b)=C_{i \alpha} N_{\alpha k} .
$$

With the help of the relations (5) it follows that the preceding equations imply

$$
C_{i \alpha}\left[M_{\alpha \beta} T_{\beta \gamma}^{-1}(a) M_{k \gamma}-N_{\alpha \beta} T_{\beta \gamma}^{-1}(b) N_{k \gamma}\right]=0 .
$$

Since $\left|C_{i k}\right| \neq 0$ the equations (20) of the theorem follow at once. Conversely, since the matrix $\left\|M_{i k}, N_{i k}\right\|$ is of rank $n$, the equations (5) and (20) imply relations of the form (21), so that the boundary conditions of the two systems are surely equivalent.

THEOREM 8. For a self-adjoint system (16) two characteristic solutions $y_{i}(x), \bar{y}_{i}(x)$ corresponding to distinct characteristic valus $\lambda, \bar{\lambda}$ satisfy the equation

$$
\int_{a}^{b} S_{\alpha \beta}(x) y_{\alpha}(x) \bar{y}_{\beta}(x) d x=0
$$

where $S_{i k}=T_{\alpha i} B_{\alpha k}$.

This is an immediate consequence of the equation (18) and the transformation $z_{i}=T_{i \alpha} y_{\alpha}$.

For the following definition it is important to note that when the matrix $\left\|S_{i k}\right\|$ is symmetric the bilinear form $S_{\alpha \beta} f_{\alpha} \bar{f}_{\beta}$, formed for a set of numbers $f_{i}$ and their conjugate imaginaries $\bar{f}_{i}$, is always real, since such a form is identical with its conjugate. The functions $g_{\alpha}(x)$ in the definition are supposed to be continuous on the interval $a b$.

Definition of a definitely self-adjoint boundary value problem. A problem (16) is said to be definitely self-adjoint if the matrix $\left\|S_{i k}(x)\right\|$ is symmetric and the bilinear form $S_{\alpha \beta}(x) f_{\alpha} \bar{f}_{\beta}$ is positive or zero at every point of the interval $a b$, and if furthermore this form vanishes identically for a set of solutions $f_{i}(x)$ of a system of equations of the type

$$
f_{i}^{\prime}(x)=A_{i \alpha}(x) f_{\alpha}(x)+B_{i \alpha}(x) g_{\alpha}(x)
$$

only when the functions $f_{i}(x)$ are all identically zero.

The conditions of the definition will surely be satisfied if the quadratic form $S_{\alpha \beta}(x) f_{\alpha} f_{\beta}$ is positive definite at every point of the interval $a b$, since the bilinear form $S_{\alpha \beta} f_{\alpha} \bar{f}_{\beta}$ is then always positive for non-vanishing arguments $f_{i}$, as one readily verifies. In that case the determinant $\left|S_{i k}\right|=\left|T_{i k}\right|\left|B_{i k}\right|$ is 
everywhere different from zero. The same is therefore true of $\left|B_{i k}\right|$, and the second equation (19) shows that

$$
0=S_{i k}-S_{k i}=T_{\alpha i} B_{\alpha k}-T_{\alpha k} B_{\alpha i}=\left(T_{\alpha i}+T_{i \alpha}\right) B_{\alpha k} .
$$

It follows readily that the matrix $\left\|T_{i k}\right\|$ is skew-symmetric. Since a skewsymmetric determinant of odd order always vanishes, and since the determinant $\left|T_{i k}\right|$ must be different from zero, it is clear that this case can arise only when $n$ is even. It should be noted, however, that the definition is applicable to cases when the determinant $\left|B_{i k}\right|$ vanishes and the quadratic form $S_{\alpha \beta} f_{\alpha} f_{\beta}$ is not definite. Important special cases of this sort are the boundary value problems arising from the calculus of variations and the problem which arises when a boundary value problem for a self-adjoint linear differential equation of the $n$th order, of a type hitherto often studied,* is transformed into one of the type (16).

If the bilinear form $S_{\alpha \beta} f_{\alpha} \bar{f}_{\beta}$ is non-positive it can always be replaced by one which is non-negative by using the transformation with coefficients $-T_{i k}$ instead of $T_{i k}$. The requirement of symmetry for the matrix $\left\|S_{i k}\right\|$ is also not as stringent as it perhaps appears to be at first sight. If the equations (19) have a system of solutions $T_{i k}$ then the systems $\bar{T}_{i k}=T_{k i}$ and $\bar{T}_{i k}-T_{i k}$ are also solutions. The matrix of elements $\bar{T}_{i k}-T_{i k}=T_{k i}-T_{i k}$ is skew-symmetric, and one readily verifies by means of the relations (19) that for a skew-symmetric system $T_{i k}$ the elements $S_{i k}$ have the symmetry required. If the matrix of elements $T_{k i}-T_{i k}$ is to be useful for a transformation, however, the determinant $\left|T_{k i}-T_{i k}\right|$ must be different from zero.

When the problem (16) is definitely self-adjoint the elements $S_{i k}$ are expressible in the various forms

$$
S_{i k}=T_{\alpha i} B_{\alpha k}=T_{\alpha k} B_{\alpha i}=-T_{i \alpha} B_{\alpha k}
$$

as one readily verifies from the symmetry of $S_{i k}$ and the relations (19).

3. Properties of self-adjoint systems. Boundary value problems of the type (16) which are definitely self-adjoint have many properties analogous to those of linear integral equations with symmetric kernel functions, as indicated in the following theorems.

TheORem 9. For a definitely self-adjoint boundary value problem (16) all roots of the characteristic determinant $D(\lambda)$ are real and the linearly independent characteristic solutions corresponding to each root may be chosen real.

- See, for example, Darboux, Théorie des Surfaces, vol. 2, p. 109; Birkhoff, loc. cit., p. 373; Bounitzky, p. 88. 
For suppose $\lambda$ a root of $D(\lambda)$ and $y_{i}(x)$ a non-vanishing solution of the system (16) corresponding to it, and let $\bar{\lambda}$ and $\bar{y}_{i}(x)$ be their conjugate imaginaries. If $\lambda$ were not real, equation (22) of Theorem 8 would require the bilinear form $S_{\alpha \beta} y_{\alpha} \bar{y}_{\beta}$ to vanish identically in $x$, which is impossible when the solution $y_{i}(x)$ is not identically zero. Hence the root $\lambda$ is real. But if $\lambda$ is real then the real and imaginary parts of $y_{i}(x)$ are separately solutions of the system (16), and it is evident that a linearly independent set of real characteristic solutions corresponding to $\lambda$ can be selected.

THEOREM 10. The index of each characteristic number $\lambda_{0}$, i.e., the number of linearly irdependent characteristic solutions $y_{i}(x)$ corresponding to it, is equal to the multiplicity of $\lambda_{0}$ as a root of $D(\lambda)$.

Suppose that $D(\lambda)=\left|s_{i}\left[Y_{k}(x, \lambda)\right]\right|$ has rank $n-r$ at a particular value $\lambda_{0}$. By replacing the solutions $Y_{k}(x, \lambda)$ by suitably selected linear combinations of them with constant coefficients it may be brought about that for $\lambda=\lambda_{0}$ the expressions $s_{i}\left(Y_{p}\right)(p=1, \cdots, r)$ all vanish, while the matrix of elements $s_{i}\left(Y_{q}\right)(q=r+1, \cdots, n)$ has rank $n-r$. All derivatives of $D(\lambda)$ of order less than $r$ will then clearly vanish at $\lambda=\lambda_{0}$, and the $r$ th will have the value

$$
D^{(r)}\left(\lambda_{0}\right)=\left|s_{i}\left(Y_{1 \lambda}\right), \cdots, s_{i}\left(Y_{r \lambda}\right), s_{i}\left(Y_{r+1}\right), \cdots, s_{i}\left(Y_{n}\right)\right|
$$

where the subscript $\lambda$ indicates derivatives. If this expression vanished there would be a linear combination

$$
y_{i}=\left(c_{1} Y_{i 1 \lambda}+\cdots+c_{r} Y_{i r \lambda}\right)+\left(c_{r+1} Y_{i, r+1}+\cdots+c_{n} Y_{i n}\right)
$$

for which all the numbers $s_{i}(y)$ would vanish at $\lambda=\lambda_{0}$. The constants $c_{1}, \cdots, c_{r}$ could not all be zero because the rank of the last $n-r$ columns of $D^{(r)}\left(\lambda_{0}\right)$ is $n-r$. The functions $y_{i 1}$ whose derivatives for $\lambda$ are in the first parenthesis would therefore not vanish identically. For $\lambda=\lambda_{0}$ they would satisfy the system (16) and also the equations

$$
y_{i 1 \lambda}^{\prime}=\left(A_{i \alpha}+\lambda B_{i \alpha}\right) y_{\alpha 1 \lambda}+B_{i \alpha} y_{\alpha 1} \text {. }
$$

The set $y_{i 2}$ defined by the second parenthesis would satisfy the differential equations of the system (16), and it follows readily that the functions $y_{i}$ themselves would for $\lambda=\lambda_{0}$ be solutions of the non-homogeneous system

$$
y_{i}^{\prime}=\left(A_{i \alpha}+\lambda_{0} B_{i \alpha}\right) y_{\alpha}+B_{i \alpha} y_{\alpha 1}, \quad s_{i}(y)=0 \text {. }
$$

The functions $z_{i 1}=T_{i \alpha} y_{\alpha 1}$ would satisfy the adjoint equations (17), and from equations (13) of Theorem 4 it would follow that

$$
\int_{a}^{b} z_{\alpha 1} B_{\alpha \beta} y_{\beta 1} d x=\int_{a}^{b} y_{\alpha 1} S_{\alpha \beta} y_{\beta 1} d x=0 .
$$


This could not be true, however, since the functions $y_{i 1}$ would not all vanish, as was seen above. It follows therefore that the derivative (25) is different from zero and that $\lambda_{0}$ has its multiplicity equal to its index.

THEOREY 11. For a system of functions $f_{i}(x)$ continuous on the interval ab and satisfying the condition

$$
\int_{a}^{b} y_{\alpha}(x) S_{\alpha \beta}(x) f_{\beta}(x) d x=0
$$

with every characteristic solution $y_{i}(x)$ of the boundary value problem (16), the functions $B_{i \alpha}(x) f_{\alpha}(x)$ all vanish identically.*

To prove this let $f_{i}(x)$ be a set with the properties described in the theorem. According to Theorems 3 and 4 the non-homogeneous system

$$
y_{i}^{\prime}=\left(A_{i \alpha}+\lambda B_{i \alpha}\right) y_{\alpha}+B_{i \alpha} f_{\alpha}, \quad s_{i}(y)=0
$$

then has solutions for every value of $\lambda$, since the equations (26) imply the conditions analogous for this case to equations (13) of Theorem 4 . When $D(\lambda) \neq 0$ there is a unique solution and one verifies readily that it consists of the functions

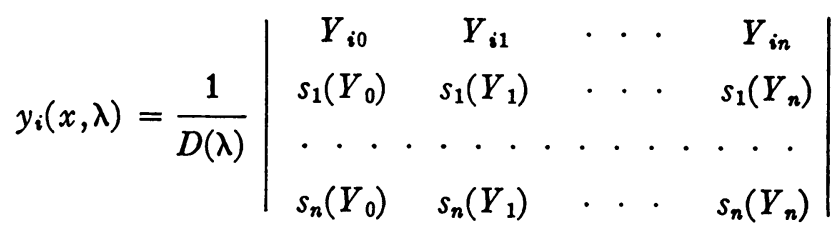

where $Y_{i 0}(x, \lambda)$ is a particular solution of the differential equations in the system (27).

Near a root $\lambda_{0}$ of multiplicity $r$ of $D(\lambda)$ the functions $y_{i}(x, \lambda)$ are still welldefined and analytic in $\lambda$. For, in the first place, one can add constant multiples of the last $n$ columns of the determinant to the first column in such a way that the resulting functions $Y_{i 0}$ satisfy the conditions $s_{i}\left(Y_{0}\right)=0$ at $\lambda=\lambda_{0}$. In the second place the $r$ linearly independent solutions $z_{i}(x)$ of the adjoint system (17) for $\lambda=\lambda_{0}$ provide $r$ linearly independent sets $\bar{t}_{\alpha}(z)$, as in the proof of Theorem 2, such that

$$
s_{\alpha}\left(Y_{1}\right) \bar{t}_{\alpha}(z)=\cdots=s_{\alpha}\left(Y_{n}\right) \bar{t}_{\alpha}(z)=0 .
$$

One can therefore replace $r$ of the rows of the determinant in the expressions (28) by $r$ linear combinations of its $n$ rows which vanish at $\lambda=\lambda_{0}$.

* The idea underlying the proof of this theorem is very well known. It has been used, for example, by E. Schmidt, p. 18; Hurwitz, p. 539; and Camp, p. 37. 
It is clear that the determinant in (28) has the same factor $\left(\lambda-\lambda_{0}\right)^{r}$ as $D(\lambda)$, and hence that the functions $y_{i}(x, \lambda)$ are analytic near $\lambda_{0}$ as well as near the values of $\lambda$ at which $D(\lambda) \neq 0$.

The functions $y_{i}(x, \lambda)$ are representable by permanently convergent power series in $\lambda$ of the form

$$
y_{i}(x, \lambda)=u_{i 0}(x)+u_{i 1}(x) \lambda+u_{i 2}(x) \lambda^{2}+\cdots .
$$

By substituting these series in the equations (27) and comparing coefficients of $\lambda$ it is found that the coefficients $u_{i \mu}(\mu=0,1,2, \cdots)$ satisfy the equations

$$
u_{i \mu}^{\prime}=A_{i \alpha} u_{\alpha \mu}+B_{i \alpha} u_{\alpha, \mu-1}, \quad s_{i}\left(u_{\mu}\right)=0
$$

in which it is agreed that $u_{i,-1}=f_{i}$. With the help of equations (30) and (19) one verifies also that the functions $v_{i}=T_{i \alpha} u_{\alpha}$ are solutions of the system

$$
v_{i v}^{\prime}=-A_{\alpha i} v_{\alpha v}-B_{\alpha i} v_{\alpha, v-1}, \quad t_{i}\left(v_{v}\right)=0,
$$

where $v_{i,-1}=T_{i \alpha} f_{\alpha}$, and it follows that

$$
u_{\alpha \mu \nu}^{\prime} v_{\alpha \nu}+u_{\alpha \mu} v_{\alpha \nu}^{\prime}=u_{\beta, \mu-1} B_{\alpha \beta} v_{\alpha \nu}-u_{\alpha \mu} B_{\beta \alpha} v_{\beta, v-1} \text {. }
$$

This result and the equations (7) and (24) now justify the relation

$$
0=\left.u_{\alpha \mu} v_{\alpha \nu}\right|_{a} ^{b}=\int_{a}^{b} u_{\alpha, \mu-1} S_{\alpha \beta} u_{\beta \nu} d x-\int_{a}^{b} u_{\alpha \mu} S_{\alpha \beta} u_{\beta, \nu-1} d x .
$$

The analogue of a well known inequality of Schwarz

$$
\left(\int_{a}^{b} u_{\alpha, \mu-1} S_{\alpha \beta} u_{\alpha, \mu+1} d x\right)^{2} \leqq \int_{a}^{b} u_{\alpha, \mu-1} S_{\alpha \beta} u_{\alpha, \mu-1} d x \int_{a}^{b} u_{\alpha, \mu+1} S_{\alpha \beta} u_{\alpha, \mu+1} d x,
$$

which will be proved in the next section, together with the last equation, show that the constants

have the properties

$$
W_{\mu}=\int_{a}^{b} u_{\alpha 0} S_{\alpha \beta} u_{\alpha \mu} d x
$$

$$
W_{\mu+\nu}=\int_{a}^{b} u_{\alpha \mu} S_{\alpha \beta} u_{\alpha \nu} d x, \quad W_{2 \mu-2} W_{2 \mu+2} \geqq W_{2 \mu}^{2} .
$$

The series

$$
W_{0}+W_{1} \lambda+\cdots, \quad W_{0}+W_{2} \lambda^{2}+\cdots,
$$

the first of which is found by integrating the expression $u_{\alpha 0} S_{\alpha \beta} y_{\beta}$ found from (29), converge for every value of $\lambda$. Some coefficient in the second series must vanish. Otherwise it would follow from the inequality (31) that 
$W_{24} \geqq W_{0}\left(W_{2} / W_{0}\right)^{\mu}$, and the second series (32) would not converge for $\lambda=$ $\left(W_{2} / W_{0}\right)^{3}$. But if a particular coefficient $W_{2 \mu}$ vanishes the inequalities (31) imply that all the preceding ones of even index are also zero. From the equation $W_{0}=0$ it follows, however, that the functions $u_{i 0}$ all vanish identically, and the first of the equations (30) shows that the functions $u_{i_{-}-1}=B_{i a} f_{a}$ do the same.

Corollary 1. If the determinant $\left|B_{i k}(x)\right|$ is different from zero on the interval ab, as in the case when the matrix $\left\|S_{i k}\right\|=\left\|T_{\alpha i} B_{\alpha k}\right\|$ is positive definite at each point of $a b$, then the set of functions $f_{i}(x)=0$ is the only one satisfying the relation (26) with all characteristic solutions $y_{i}(x)$ of the boundary value problem (16).

COROLLARY 2. The only set of solutions $f_{i}(x)$ of a system of equations of the form

$$
f_{i}^{\prime}=A_{i \alpha} f_{\alpha}+B_{i \alpha} g_{\alpha}
$$

satisfying the relations (26) with all characteristic solutions $y_{i}(x)$ is the set $f_{i}(x) \equiv 0$.

With the help of the last corollary it is possible to prove

THEOREM 12. The totality of characteristic solutions and characteristic constants of the boundary value problem (16) are denumerably infinite in number and may be represented by the symbols $y_{i v}(x), \lambda_{p}(\nu=1,2, \cdots)$. These functions may furthermore be chosen normed and orthogonal in the sense that

$$
\int_{a}^{b} y_{\alpha \mu} S_{\alpha \beta} y_{\beta \nu} d x=\delta_{\mu \nu} \quad\left(\delta_{\mu \mu}=1, \delta_{\mu \nu}=0 \text { if } \mu \neq \nu\right) .
$$

Let the functions $g_{\alpha}$ in equations (33) have the form $g_{i}=d_{p} g_{i p}(\rho=1, \cdots$, $p)$. The functions $g_{i p}$ can be chosen so that the $p$ systems $B_{i \alpha} g_{\alpha p}(\rho=1, \ldots$, $p$ ) are linearly independent, since the functions $B_{i k}$ do not all vanish identically. The system (33) has a solution of the form $f_{i}=d_{p} f_{i p}$ where the system $f_{i p}$ for $p$ fixed is a particular solution of the equations (33) with $g_{i}$ replaced by $g_{i p}$. The solutions $f_{i p}$ are readily seen to be linearly independent since the sets $B_{i \alpha} g_{\alpha \rho}(\rho=1, \cdots, p)$ have this property. If there were a finite number only of characteristic solutions of the boundary value problem (16) then for a sufficiently large value of $p$, constants $d_{\rho}$ not all zero could always be selected so that the functions $f_{i}=d_{p} f_{i p}$ would satisfy the relations (26) with every such solution, and this would contradict Corollary 2 above. Hence there must be an infinity of characteristic solutions, and the infinity is denumerable since the roots of the permanently convergent series $D(\lambda)$ are denumerable. 
Since no one of the sets $y_{i \mu}(x)$ for fixed $\mu$ is identically zero the integrals (26) for $\nu=\mu$ are all different from zero. The characteristic functions $y_{i \mu}(x)$ may therefore be normed and orthogonalized as described in the theorem by a well known process.*

4. Expansion theorems. With the help of the theorems of the last section it is possible to deduce some very general expansion theorems for sets of functions $f_{i}(x)$. The characteristic solutions $y_{i \mu}(x)$ of the definitely self-adjoint boundary value problem (16), appearing in these theorems, are supposed to be normed and orthogonal.

THEOREM 13. For every solution $f_{\dot{i}}(x)$ of a system of equations of the form

$$
f_{i}^{\prime}=A_{i \alpha} f_{\alpha}+B_{i \alpha} g_{\alpha}, \quad s_{i}(f)=0,
$$

in which the functions $g_{i}(x)$ are arbitrarily selected continuous functions on the interval ab, the series

$$
\varphi_{i}(x)=\sum_{\nu=1}^{\infty} y_{i \nu}(x) \int_{a}^{b} y_{\alpha \nu}(\xi) S_{\alpha \beta}(\xi) f_{\beta}(\xi) d \xi
$$

converge uniformly and the functions $B_{i \alpha}\left(f_{\alpha}-\varphi_{\alpha}\right)$ are all identically zero on ab.

The uniform convergence of the series will be proved in a later section. The rest of the theorem follows at once from Theorem 11 since the equations

$$
\int_{a}^{b} y_{\alpha \mu} S_{\alpha \beta}\left(f_{\beta}-\varphi_{\beta}\right) d x=0 \quad(\mu=1,2, \cdots)
$$

are immediate consequences of the definition of the functions $\varphi_{i}$.

Corollary 1. If the determinant $\left|B_{i k}(x)\right|$ is different from zero on the interval ab, as in the case when the matrix $\left\|S_{i k}\right\|=\left\|T_{\alpha i} B_{\alpha k}\right\|$ is positive definite at each point of $a b$, then for every set of functions $f_{i}(x)$ having continuous derivatives on this interval and satisfying the boundary conditions $s_{i}(f)=0$ the series (36) converge uniformly and represent the functions $f_{i}(x)$.

In this case the equations (35) determine uniquely a set of functions $g_{i}(x)$ corresponding to the given functions $f_{i}(x)$, since the determinant $\left|B_{i k}\right|$ is different from zero.

COROLlaRy 2. If the functions $f_{i}(x)$ are solutions of a system (35) and the functions $g_{i}(x)$ solutions of a similar system

$$
g_{i}{ }^{\prime}=A_{i \alpha} g_{\alpha}+B_{i \alpha} h_{\alpha}, \quad s_{i}(g)=0,
$$

\footnotetext{
* See, for example, E. Schmidt, p. 4.
} 
in which the functions $h_{i}(x)$ are continuous on ab, then the series (36) converge uniformly and represent the functions $f_{i}(x)$ on this interval.

To prove this the equations (35), (17), and (7) can first be used to show that for every characteristic solution $y_{i v}(x)$ of the system (15) the corresponding functions $z_{i \nu}=T_{i \alpha} y_{\alpha}$ satisfy the relations

$$
\begin{gathered}
z_{\alpha \nu} f_{\alpha}^{\prime}+z_{\alpha \nu}^{\prime} f_{\alpha}=z_{\alpha \nu} B_{\alpha \beta} g_{\beta}-\lambda_{\nu} z_{\beta \nu} B_{\beta \alpha} f_{\alpha}, \\
0=\left.z_{\alpha \nu} f_{\alpha}\right|_{a} ^{b}=\int_{a}^{b} y_{\alpha \nu} S_{\alpha \beta} g_{\beta} d x-\lambda, \int_{a}^{b} y_{\alpha \nu} S_{\alpha \beta} f_{\beta} d x .
\end{gathered}
$$

Since the functions $g_{i}(x)$ satisfy equations (37) it will be seen that the series

$$
\sum, \lambda_{\nu} y_{i \nu}(x) \int_{a}^{b} y_{\alpha \nu} S_{\alpha \beta} f_{\beta} d \xi=\sum, y_{i \nu}(x) \int_{a}^{b} y_{\alpha} S_{\alpha \beta} g_{\beta} d \xi
$$

converges uniformly by the same proof as that which shows the convergence of the series (36). It follows readily with the help of the differential equations (16) for the functions $y_{i}(x)$ that the series of derivatives of the terms of (36) converges uniformly and represents $\varphi_{i}^{\prime}(x)$. Further

$$
\varphi_{i}^{\prime}(x)=A_{i \alpha} \varphi_{\alpha}+B_{i \alpha} \sum_{\nu} y_{\alpha \nu}(x) \int_{a}^{b} y_{\alpha \nu} S_{\alpha \beta} g_{\beta} d \xi .
$$

Since the functions $B_{i \alpha}\left(f_{\alpha}-\varphi_{\alpha}\right)$ vanish identically, $\left(f_{\alpha}-\varphi_{\alpha}\right) S_{\alpha \beta}\left(f_{\beta}-\varphi_{\beta}\right)$ vanishes identically. Since from equations (35) and (39) the differences $f_{\alpha}-\varphi_{\alpha}$ satisfy an equation of the type (23) it follows that they are identically zero, which was to be proved.

5. Green's matrix. Consider again the system

$$
y_{i}^{\prime}=A_{i \alpha} y_{\alpha}, \quad s_{i}(y)=M_{i \alpha} y_{\alpha}(a)+N_{i \alpha} y_{\alpha}(b)=0
$$

described in $\S 1$, and suppose that its determinant $D$ is different from zero.

Definition of the Green's matrix.* A matrix of functions $G_{i k}(x, \zeta)$, single-valued for $a \leqq x \leqq b, a \leqq \xi \leqq b$, and further such that they are continuous and have continuous first derivatives in $x$ except at $x=\xi$, is called the Green's matrix of the system (16) if it has the properties

$$
\begin{aligned}
& G_{i k}(\xi+0, \xi)-G_{i k}(\xi-0, \xi)=\delta_{i k} \quad\left(\delta_{i i}=1, \delta_{i k}=0 \text { if } i \neq k\right), \\
& \frac{\partial}{\partial x} G_{i k}(x, \xi)=A_{i \alpha}(x) G_{\alpha k}(x, \xi), \\
& M_{i \alpha} G_{\alpha k}(a, \xi)+N_{i \alpha} G_{\alpha k}(b, \xi)=0 .
\end{aligned}
$$

* The existence and uniqueness of the Green's matrix has been proved by several writers. See Bounitzky, p. 77; Birkhoff-Langer, pp.66-70. 
The properties (42) and (43) show that the columns of the matrix would be solutions of the system (16) of the kind demanded by the boundary value problem if it were not for their discontinuities at $x=\xi$. Let $D_{i k}$ and $\Delta_{i k}$ be defined by the equations

$$
D_{i k}=M_{i \alpha} Y_{\alpha k}(a)+N_{i \alpha} Y_{\alpha k}(b), \quad \Delta_{i k}=M_{i \alpha} Y_{\alpha k}(a)-N_{i \alpha} Y_{\alpha k}(b) .
$$

The determinant $D=\left|D_{i k}\right|$ is different from zero by hypothesis. The functions

$$
G_{i k}(x, \xi)=\frac{1}{2} Y_{i \alpha}(x)\left[\frac{|x-\xi|}{x-\xi} \delta_{\alpha \gamma}+D_{\alpha \beta}^{-1} \Delta_{\beta \gamma}\right] Y_{\gamma k}^{-1}(\xi)
$$

are then well-defined and evidently have the properties (41) and (42). Equation (43) follows readily with the help of the notations (44).

THEOREM 14. The functions $H_{i k}(x, \xi)=-G_{k i}(\xi, x)$ defined by the equations (45) are the elements of the Green's matrix of the adjoint system (17), so that

$$
\begin{gathered}
G_{k i}(x, x-0)-G_{k i}(x, x+0)=\delta_{k i}, \\
\frac{\partial}{\partial \xi} G_{k i}(x, \xi)=-A_{\alpha i}(\xi) G_{k \alpha}(x, \xi), \\
P_{\alpha i} G_{k \alpha}(x, a)+Q_{\alpha i} G_{k \alpha}(x, b)=0 .
\end{gathered}
$$

By differentiating the equations $Y_{i \alpha} Y_{\alpha k}^{-1}=\delta_{i k}$ it can be shown that the rows of the matrix $Y_{\mathbf{a}}^{-1}$ are solutions of the differential equations of the adjoint system (17), and the properties (46) and (47) are then evident from the form of the equations (45). The relations $M_{i a} P_{\alpha k}-N_{i a} Q_{a k}=0$ between the elements of the reciprocal matrices (6) justify the equations

$$
\Delta_{i \alpha}\left[P_{\beta k} Y_{\alpha \beta}^{-1}(a)+Q_{\alpha i} Y_{\alpha \beta}^{-1}(b)\right]=D_{i \alpha}\left[-P_{\beta k} Y_{\alpha \beta}^{-1}(a)+Q_{\beta k} Y_{\alpha \beta}^{-1}(b)\right]
$$

and equation (48) can be proved with the help of them.

TheOREM 15. If the functions $g_{i}(x)$ are continuous on the interval $a b$ then every solution $y_{i}(x)$ of the non-homogeneous system

$$
y_{i}^{\prime}=A_{i \alpha} y_{\alpha}+g_{i}, \quad s_{i}(y)=0
$$

is expressible in the form

$$
y_{i}(x)=\int_{a}^{b} G_{i \alpha}(x, \xi) g_{\alpha}(\xi) d \xi,
$$

and conversely every system $y_{i}(x)$ defined by equations (50) is a solution of (49). 
From the equations (49) for $x=\xi$, and equations (47), it follows that

Hence

$$
\frac{\partial}{\partial \xi}\left[G_{k \alpha}(x, \xi) y_{\alpha}(\xi)\right]=G_{k \alpha}(x, \xi) g_{\alpha}(\xi) \text {. }
$$

$$
\begin{aligned}
& G_{k \alpha}(x, x-0) y_{\alpha}(x)-G_{k \alpha}(x, a) y_{\alpha}(a)=\int_{a}^{x} G_{k \alpha}(x, \xi) g_{\alpha}(\xi) d \xi, \\
& G_{k \alpha}(x, b) y_{\alpha}(b)-G_{k \alpha}(x, x+0) y_{\alpha}(x)=\int_{x}^{b} G_{k \alpha}(x, \xi) g_{\alpha}(\xi) d \xi .
\end{aligned}
$$

With the help of the relations (46), (48), $s_{i}(y)=0$, and (7), the sum of these two equations gives equation (50).

The converse is easily proved by writing the expression (50) for $y_{i}(x)$ as the sum of integrals on the intervals $a x$ and $x b$, differentiating with respect to $x$, and using the relations (46) and (42).

Corollary 1. The Green's matrix is unique.

For if there were two, say $G_{i k}$ and $L_{i k}$, the equation

$$
\int_{a}^{b}\left[G_{i \alpha}(x, \xi)-L_{i \alpha}(x, \xi)\right] g_{\alpha}(\xi) d \xi=0
$$

would be an identity in $x$ for every set of functions $g_{\alpha}(\xi)$, and this can be true only if $G_{i k}=L_{i k}$.

COROLLARY 2. When $\lambda=0$ is not a characteristic number then every solution $y_{i}(x)$ of the boundary value problem

$$
y_{i}^{\prime}=\left(A_{i \alpha}+\lambda B_{i \alpha}\right) y_{i}, \quad s_{i}(y)=0
$$

is a solution of the system of linear integral equations

$$
y_{i}(x)=\lambda \int_{a}^{b} G_{i \alpha}(x, \xi) B_{\alpha \beta}(\xi) y_{\beta}(\xi) d \xi,
$$

and conversely.

By replacing $\lambda$ by $\lambda_{1}+\lambda$ in the equations (16) it can readily be brought about that the value $\lambda=0$ is not a characteristic number, if this is not already the case.

THEOREM 16. If the boundary value problem (16) is self-adjoint then the system (52) is equivalent to the corresponding system

$$
z_{i}(x)=\lambda \int_{a}^{b} z_{\alpha}(\xi) B_{\alpha \beta}(\xi) G_{\beta i}(\xi, x) d \xi
$$


for the adjoint problem by means of the transformation $z_{i}=T_{i \alpha} y_{\alpha}$. The functions $G_{i k}$ in this case satisfy the relations

$$
G_{\alpha i}(\xi, x) T_{\alpha k}(\xi)=-T_{i \alpha}(x) G_{\alpha k}(x, \xi)
$$

Since the original boundary value problem and its adjoint are equivalent under the transformation $z_{i}=T_{i \alpha} y_{\alpha}$ it is evident that the systems (52) and (53) must have the same property.

To prove equation (54) one may first deduce the second of the systems

$$
\begin{aligned}
y_{i}^{\prime} & =A_{i \alpha} y_{\alpha}+g_{\alpha}, & s_{i}(y) & =0, \\
z_{i}^{\prime} & =-A_{\alpha i} z_{\alpha}+T_{i \alpha} g_{\alpha}, & t_{i}(z) & =0
\end{aligned}
$$

from the first by means of the transformation $z_{i}=T_{i \alpha} y_{\alpha}$. From Theorem 15 it follows then that the systems

$$
\begin{aligned}
& y_{i}(x)=\int_{a}^{b} G_{i \alpha}(x, \xi) g_{\alpha}(\xi) d \xi \\
& z_{i}(x)=\int_{a}^{b} H_{i \alpha}(x, \xi) T_{\alpha \beta}(\xi) g_{\alpha}(\xi) d \xi
\end{aligned}
$$

are equivalent by this transformation. But from these one verifies that the equation

$$
\int_{a}^{b}\left[H_{i \alpha}(x, \xi) T_{\alpha \beta}(\xi)-T_{i \alpha}(x) G_{\alpha \beta}(x, \xi)\right] g_{\beta}(\xi) d \xi=0
$$

must hold for all sets of functions $g_{\alpha}$. Since $H_{i k}(x, \xi)=-G_{k i}(\xi, x)$ this proves equations (54).

If the elements of the kernel matrix of the system (52) are denoted by

$$
K_{i j}(x, \xi)=G_{i \alpha}(x, \xi) B_{\alpha j}(\xi)
$$

then this system and the one "associated" with it, according to the theory of integral equations, are

$$
\begin{aligned}
& y_{i}(x)=\lambda \int_{a}^{b} K_{i \alpha}(x, \xi) y_{\alpha}(\xi) d \xi \\
& u_{i}(x)=\lambda \int_{a}^{b} K_{\alpha i}(\xi, x) u_{\alpha}(\xi) d \xi .
\end{aligned}
$$

When the boundary value problem (51) is self-adjoint the relations (54), with (24), show that

$$
K_{\alpha i}(\xi, x) S_{\alpha k}(\xi)=S_{i \beta}(x) K_{\beta k}(x, \xi)
$$


It is provable readily then that every solution $y_{i}(x)$ of the first of equations (55) goes into a solution $u_{i}(x)$ of the second by means of the transformation $u_{i}(x)=S_{i \beta}(x) y_{\beta}(x)$. If the determinant $\left|S_{i k}\right|$ is different from zero the equations (55) are completely equivalent by means of this transformation. In that case a symmetric matrix $U_{i k}(x)$ can be determined such that

$$
U_{i \alpha}(x) U_{\alpha k}(x)=S_{i k}(x)^{*}
$$

and the transformation $v_{i}=U_{i \alpha} y_{\alpha}$ takes the first system (55) into the system

$$
v_{i}(x)=\lambda \int_{a}^{b} U_{i \alpha}(x) K_{\alpha \beta}(x, \xi) U_{\beta \gamma}^{-1}(\xi) v_{\gamma}(\xi) d \xi
$$

whose kernel matrix is readily seen to be symmetric. Such a reduction is not possible when the determinant $\left|S_{i k}\right|$ vanishes.

It is evident that every solution of the system (53) defines a solution of (55) by means of the transformation $u_{i}=B_{\alpha i} z_{\alpha}$. Conversely, if a solution $u_{i}(x)$ of the equations (55) is known, for a particular value of $\lambda$, then the functions

$$
z_{i}(x)=\lambda \int_{a}^{b} G_{\beta i}(\xi, x) u_{\beta}(\xi) d \xi
$$

satisfy the relations $u_{i}=B_{\alpha i} z_{\alpha}$ and the equations (53). If the boundary value problem (16) equivalent to the system (52) is definitely self-adjoint, every solution of the system (52) defines a solution of the conjugate system (55) by means of the symmetric transformation $u_{i}=S_{i \beta} y_{\beta}=B_{\alpha i} T_{\alpha \beta} y_{\beta}$, as one may infer from the transformation $z_{i}=T_{i \beta} y_{\beta}$ relating the solutions of the system (52) and its adjoint (53), or directly by means of the relations (54) and (24).

It is clear then that the theory of a definitely self-adjoint boundary value problem (16) may be regarded as a special case of the theory of a system of linear integral equations (52) whose solutions go over into solutions of the conjugate system (55) by means of a symmetric transformation $u_{i}=S_{i \beta} y_{\beta}$. From the preceding paragraphs it is evident that such systems have many properties analogous to those of systems whose kernel matrix is symmetric. The symmetric case is the one which arises when the matrix of functions $S_{i k}$ is the identity matrix. It would be interesting to investigate in detail the theory of such systems of linear integral equations.

6. The convergence proof. For the purpose of proving the uniform convergence of the series (36) of Section 3 a number of lemmas are required

* See, for example, Bôcher, Higher Algebra, p. 299. 
which are analogous to those used for similar purposes in the theory of a single linear integral equation, ${ }^{*}$ and which have for the most part been frequently applied in more generalized form than here given by $\mathbf{E}$. $\mathbf{H}$. Moore in his "general analysis."

It is understood that the functions $S_{i k}(x)$ are continuous and the quadratic form $S_{\alpha \beta} f_{\alpha} f_{\beta} \geqq 0$ at every $x$ on the interval $a b$ and for every set of arguments $f_{i}$. The functions $f_{i}(x), g_{\alpha}(x), y_{i \mu}(x)$ in the following theorems are at least bounded and integrable on the interval $a b$, and the sets $y_{i \mu}(x)(\mu=$ $1,2, \cdots)$ are normed and orthogonal in the sense described in Theorem 10. The constants $\gamma_{\mu}$ are the numbers

$$
\gamma_{\mu}=\int_{a}^{b} f_{\alpha}(x) S_{\alpha \beta}(x) y_{\beta \mu}(x) d x .
$$

The first lemma below follows at once from the readily proved equation

$$
\int_{a}^{b}\left[f_{\alpha}-\sum \gamma_{\mu} y_{\alpha \mu}\right] S_{\alpha \beta}\left[f_{\beta}-\sum \gamma_{\nu} y_{\beta}\right] d x=\int_{a}^{b} f_{\alpha} S_{\alpha \beta} f_{\beta} d x-\sum \gamma_{\mu}^{2},
$$

where the sums without range indicated are taken with respect to $\mu, \nu$ over the same arbitrarily selected set of a finite number of positive integers. The second and third lemmas are immediate consequences of the first one. The integral in the second member of equation (56) is called the norm of the set $f_{i}(x)$.

LEMMA 1. $\quad \sum \gamma_{\mu}^{2}<\int_{a}^{b} f_{\alpha} S_{\alpha \beta} f_{\beta} d x$.

LEMMA 2. $\quad \sum_{\mu=1}^{\infty} \gamma_{\mu}^{2}$ converges.

LEMMA 3. If the norm of the set $g_{i}(x)$ is zero then

$$
\int_{a}^{b} f_{\alpha} S_{\alpha \beta} g_{\beta} d x \leqq \int_{a}^{b} f_{\alpha} S_{\alpha \beta} f_{\beta} d x \int_{a}^{b} g_{\alpha} S_{\alpha \beta} g_{\beta} d x .
$$

Suppose now that the functions $h_{i}(x, \xi)$ are bounded for all values of $x$ and $\xi$ on the interval $a b$, and integrable in $x$ on that interval for every fixed $\xi$. Let $\delta_{\mu}$ and $\epsilon_{\mu}(\xi)$ denote the integrals

$$
\delta_{\mu}=\int_{a}^{b} g_{\alpha} S_{\alpha \beta} y_{\beta \mu} d x, \quad \epsilon_{\mu}(\xi)=\int_{a}^{b} h_{\alpha}(x, \xi) S_{\alpha \beta}(x) y_{\beta \mu}(x) d x .
$$

* See E. Schmidt, pp. 1-4. 
LEMMA 4. The series $\sum_{\mu=1}^{\infty} \delta_{\mu} \epsilon_{\mu}(\xi)$ converges uniformly on the interval $a \leqq \xi \leqq b$.

The proof is analogous to one given by Schmidt.* In the sum

$$
\sum_{\mu=p}^{p+q}\left|\delta_{\mu} \epsilon_{\mu}(\xi)\right|=\sum \delta_{\rho} \epsilon_{\rho}(\xi)-\sum \delta_{\sigma} \epsilon_{\sigma}(\xi)
$$

let the index $\rho$ range over those numbers for which $\delta_{\rho} \epsilon_{\rho}(\xi)$ is positive for a fixed $\xi$, and $\sigma$ over those for which these terms are negative. Then by Lemma 3

$$
\begin{aligned}
\sum \delta_{\rho} \epsilon_{\rho}(\xi) & =\int_{a}^{b} h_{\alpha}(x, \xi) S_{\alpha \beta}(x) \sum \delta_{\rho} y_{\beta \rho}(x) d x \\
& \leqq\left[\int_{a}^{b} h_{\alpha} S_{\alpha \beta} h_{\beta} d x \cdot \sum \delta_{\mu}^{2}\right]^{1 / 2} \\
& \leqq A^{1 / 2}\left(\sum_{\mu=D}^{\infty} \delta_{\mu}^{2}\right)^{1 / 2},
\end{aligned}
$$

where $A$ is the maximum of the norm of the functions $h_{\alpha}(x, \xi)$ on the interval $a \leqq \xi \leqq b$. A similar inequality holds for the negative terms, and it follows that

$$
\sum_{\mu=p}^{p+n}\left|\delta_{\mu} \epsilon_{\mu}(\xi)\right| \leqq 2 A^{1 / 2}\left(\sum_{\mu=p}^{\infty} \delta_{\mu}^{2}\right)^{1 / 2} .
$$

Since the series of Lemma 2 converges it is evident that the similar series of terms $\delta_{\mu}^{2}$ has the same property, and from the last inequality it follows that the series of Lemma 4 converges uniformly on the interval $a b$.

THEOREM 17. If the functions $f_{i}(x)$ are solutions of a system of the form

$$
f_{i}^{\prime}=A_{i \alpha} f_{\alpha}+B_{i \alpha} g_{\alpha}, \quad s_{i}(f)=0
$$

then the series $\sum_{\mu=1}^{\infty} \gamma_{\mu} y_{i \psi}(x)$ converges uniformly on the interval $a b$.

From equations (38) and (52) it follows that the series of the theorem are also expressible in the form

$$
\begin{aligned}
\sum_{\mu=1}^{\infty} \gamma_{\mu} y_{i \mu}(x) & =\sum_{\mu=1}^{\infty} \frac{1}{\lambda_{\mu}} y_{i \mu}(x) \int_{a}^{b} g_{\alpha} S_{\alpha \beta} y_{\beta \mu} d x \\
& =\sum_{\mu=1}^{\infty} \delta_{\mu} \int_{a}^{b} G_{i \alpha}(x, \xi) T_{\alpha \beta}^{-1}(\xi) S_{\beta \gamma}(\xi) y_{\gamma \mu}(\xi) d \xi .
\end{aligned}
$$

\footnotetext{
* Pp. 2-4.
} 
For each fixed value of $i$ this is a series of the form whose uniform convergence is stated in Lemma 4.

\section{A List OF REFERENCES}

(1) E. Schmidt, Entwickelung willkürlicher Funktionen nach Systemen vorgeschriebener. Dissertation, Göttingen, 1905.

(2) Bounitzky, Journal de Mathématiques, ser. 6, vol. 5 (1909), p. 65.

(3) Hildebrandt, these Transactions, vol. 19 (1918), p. 94.

(4) E. H. Moore, Bulletin of the American Mathematical Society, vol. 18 (1912), p. 334.

(5) Schur, Mathematische Annalen, vol. 72 (1921), p. 213.

(6) Hurwitz, these Transactions, vol. 22 (1921), p. 526.

(7) Camp, American Journal of Mathematics, vol. 44 (1922), p. 25.

(8) Carmichael, American Journal of Mathematics, vol. 43 (1921), pp. 69, 232; vol. 44 (1922), p. 129; Bulletin of the American Mathematical Society, vol. 28 (1922), p. 200.

(9) Birkhoff-Langer, Proceedings of the American Academy of Arts and Sciences, vol. 58 (1923), p. 51. See also their abstract, Bulletin of the American Mathematical Society, vol. 27 (1922), p. 236.

Untversity of Chicago, Chrcago, Irr. 